Mirai. Estudios Japoneses

ISSN-e: 2531-145X

https://dx.doi.org/10.5209/mira.74697

\title{
La diplomacia olímpica de Japón: De Tokio 1964 a Tokio 2021
}

\author{
Juan Luis López Aranguren ${ }^{1}$
}

Fecha de recepción: 31/01/2021/ Fecha de aceptación: 11/04/2021

Resumen: La diplomacia pública o cultural es el intercambio de cultura, ideas e información que las naciones de todo el mundo realizan para lograr una comprensión mutua que permita avanzar en la construcción de un mundo más pacífico y estable. En este ámbito, la celebración de los Juegos Olímpicos es uno de los eventos de diplomacia pública o cultural más importante que una nación puede lograr para proyectar y compartir su cultura e identidad con el resto del mundo. La hipótesis a confirmar es que Japón, como país democrático que protege la libertad de expresión, logra una diplomacia cultural más efectiva porque integra la participación de una gran variedad de actores culturales de toda la sociedad nacional japonesa que contribuyen a fortalecer el mensaje cultural japonés. Esta investigación estudiará las conexiones entre la sociedad japonesa, la comunicación internacional y la protección jurídica de la libertad de expresión, con el objetivo de ayudar a los actores culturales, académicos, políticos y comunicativos que desean mejorar y fortalecer tanto la diplomacia cultural japonesa como la llamada diplomacia olímpica en el resto del mundo. Para alcanzar los objetivos anteriormente expuestos, a través de una investigación comparativa e interdisciplinar se identificará y analizará la evolución de la diplomacia cultural de Japón desde los Juegos Olímpicos de Tokio de 1964 hasta los JJOO de 2021. Esta investigación también permitirá comprender la futura diplomacia cultural de Japón después de la era postolímpica y permitirá analizar el rol de Japón como una potencia cultural en el siglo XXI.

Palabras clave: Japón, Diplomacia pública, Diplomacia olímpica, poder blando, relaciones internacionales.

\section{[en] Japan's Olympic Diplomacy: From Tokyo 1964 to Tokyo 2021}

\begin{abstract}
Public or cultural diplomacy is the exchange of culture, ideas and information that nations around the world carry out to achieve mutual understanding that allows progress in the construction of a more peaceful and stable world. In this area, the celebration of the Olympic Games is one of the most important events of public or cultural diplomacy that a nation can achieve to project and share its culture and identity with the rest of the world. The hypothesis to be tested is that Japan, as a democratic country that protects freedom of expression, achieves a more effective cultural diplomacy because it integrates the participation of a great variety of cultural actors. This research will study the connections between Japanese society, international communication and the legal protection of freedom of expression. To achieve the aforementioned objectives, a comparative and interdisciplinary research will be carried out analyzing the evolution of Japan's cultural diplomacy from the 1964 Tokyo Olympics to the 2021 Olympic Games. This research will also allow to understand future cultural diplomacy of Japan after the post-Olympic era and will allow the analysis of the role of Japan as a cultural power in the 21 st century.
\end{abstract}

Keywords: Japan, Olympic Diplomacy, Public Diplomacy, Soft Power, International Relations.

Sumario: 1. Introducción. 2. Los JJ. OO. de Tokio como máximo exponente de la diplomacia pública de Japón. a) Diplomacia pública o cultural. b) Poder blando o soft power. 3. Japón como anfitriona de los JJ. OO.: De Tokio 1964 a Tokio 2021. 3.1. La diplomacia pública de los Juegos Olímpicos de Tokio de 1964. 3.2. La diplomacia pública de los Juegos Olímpicos de Tokio de 2021. 3.2.1 Primer objetivo: fortalecer la conexión entre la ciudadanía y los valores olímpicos. 3.2.2. Segundo objetivo: reforzar la proyección cultural de Japón. 3.2.3 Tercer objetivo: asegurar un legado a futuras generaciones. 4. Metodología: Indicadores internacionales de la diplomacia pública de Japón. 5. Conclusiones. 6. Bibliografía.

Cómo citar: López Aranguren, J. L. La diplomacia olímpica de Japón: De Tokio 1964 a Tokio 2021, en Mirai. Estudios Japoneses, 5 , $2021,67-76$

\section{Introducción}

Si la diplomacia tradicional se entiende como las relaciones ejercidas entre representantes oficiales de los Estados, en los últimos años ha ganado popularidad un nuevo concepto de diplomacia que se ha vuelto cada vez más importante en las relaciones entre las naciones: la diplomacia pública o cultural. Asumiendo que la cultura es el vehículo a través del cual las naciones se comunican entre sí, la diplomacia pública o cultural

\footnotetext{
Profesor de Derecho internacional público y Relaciones internacionales de la Facultad de Derecho de la Universidad de Zaragoza. Miembro del Grupo de investigación Japón. ORCID: 0000-0002-4720-863X.
}

Mirai. Estud. Japon. 5, 2021: 67-76 
es el intercambio de cultura, ideas e información que las naciones de todo el mundo realizan para lograr una comprensión mutua que permita avanzar en la construcción de un mundo más justo y estable. En este ámbito, la celebración de los Juegos Olímpicos es uno de los eventos de diplomacia pública o cultural más importante que una nación puede lograr para proyectar y compartir su cultura e identidad con el resto del mundo. En este sentido, Japón fortaleció su posición mundial como actor de diplomacia pública en la ceremonia de clausura de los JJ. OO. de Río de Janeiro de 2016. En ella, el primer ministro japonés Abe Shinzo apareció caracterizado como el personaje de videojuegos mundialmente conocido Mario para recoger el testigo de cara a los JJ. OO. de Tokio 2020 (retrasados debido a la pandemia mundial del COVID-19).

La presente investigación toma como base los Juegos Olímpicos de Tokio 2020, que previsiblemente se celebrarán en el verano de 2021, como vehículo de diplomacia pública para analizar cómo Japón comunica su identidad cultural y nacional al mundo. Para ello, se analizará esta dimensión bajo la libertad de expresión protegida por la Constitución japonesa, así como la evolución histórica de esta forma de diplomacia desde 1964 hasta 2021. La hipótesis a confirmar es que Japón, como país democrático que protege la libertad de expresión ${ }^{2}$, logra una diplomacia cultural más efectiva porque integra la participación de una gran variedad de actores culturales de toda la sociedad japonesa que contribuyen a fortalecer su mensaje cultural.

Los JJ. OO. de Tokio 2021 son una oportunidad histórica para analizar e investigar científicamente la diplomacia cultural de Japón, ya que los anteriores JJ. OO. de Tokio 1964 se celebraron en un momento en el que no había todavía una sociedad global de la información equiparable a la actual. Esta investigación estudiará las conexiones entre la sociedad japonesa, la comunicación internacional y la protección jurídica de la libertad de expresión, con el objetivo de ayudar a los actores culturales, académicos, políticos y comunicativos que, en Japón o en el resto del mundo, desean mejorar y fortalecer tanto la diplomacia cultural como la llamada diplomacia olímpica.

Para alcanzar los objetivos anteriormente expuestos, esta investigación identificará y analizará la diplomacia cultural de los Juegos Olímpicos de Tokio 2021 bajo la libertad de expresión que Japón garantiza³, comparándolos con el precedente histórico de 1964. Este estudio de caso se analizará desde dos aproximaciones diferentes: una global, donde se examinarán los Juegos Olímpicos como herramienta de diplomacia cultural en las dimensiones geopolítica y cultural empleando los índices de poder blando o diplomacia pública The Soft Power 30 y el Global Soft Power Index; y otra doméstica, donde se estudiará la libertad de expresión de Japón como un pilar constitucional de su ordenamiento legal y un rasgo distintivo de su carácter socio-político como democracia. Finalmente, esta investigación también permitirá comprender la futura diplomacia cultural de Japón en la era postolímpica y, asimismo, analizar el rol que Japón desempeña como potencia cultural en el siglo XXI.

\section{Los JJ. OO. de Tokio como máximo exponente de la diplomacia pública de Japón}

Antes de abordar la cuestión, resulta necesario acotar conceptualmente y definir dos conceptos relacionados: diplomacia pública o cultural y poder blando (o soft power). Estos dos términos aparecen en ocasiones como sinónimos para referirse al mismo tipo de acción que las diferentes naciones realizan en la dimensión comunicativa. Sin embargo, existen diferencias tanto en los matices que tiene cada uno de ellos como en la época histórica y el contexto geopolítico en el que fueron acuñados.

\section{a) Diplomacia pública o cultural}

En primer lugar, el término de diplomacia pública fue acuñado por el diplomático y decano de la Fletcher School of Law and Diplomacy, Edmund Gullion en $1965^{4}$, aunque el concepto ha existido desde siempre. Tradicionalmente, a esta práctica se la denominaba propaganda (y, de hecho, Gullion afirmó que la prefería), pero el término adquirió tal grado de connotaciones negativas que terminó siendo sustituido por diplomacia pública $^{5}$. Este término es definido por el Institute for Cultural Diplomacy (ICD) como:

Esto se ha analizado en anteriores investigaciones sobre la protección constitucional de la libertad de expresión en Japón y su impacto en el fortalecimiento de la capacidad comunicativa de la nación. Véase Juan Luis López-Aranguren, "Rational Choice Theory and International Communication: A Proposal for a New Interpretation of Article 21 of the Japanese Constitution", Osaka University Law Review 64, (Febrero 2017 ): 111-134. Sobre la protección constitucional que Japón ofrece desde una perspectiva internacional, véase Francisco Barberán, Introducción al Derecho Japonés Actual, (Pamplona, Navarra: Aranzadi 2013).

Es necesario aquí señalar el especial momento histórico en el que este desarrollo conceptual se produjo. Apenas tres años antes, en 1962, se había producido la crisis de los misiles de Cuba que puso al mundo al borde de un conflicto nuclear directo entre EEUU y la URSS en uno de los momentos más delicados de la Guerra Fría. Una posible interpretación es que esta escalada bélica obligó a los poderes mundiales a poner el énfasis en el desarrollo de metodologías que les permitieran alcanzar sus objetivos políticos sin provocar al rival, lo que explicaría la importancia de la diplomacia pública en este contexto de tensiones internacionales.

Cull, Nicholas J., "Public Diplomacy before Gullion. The Evolution of a Phrase". En Routledge Handbook of Public Diplomacy, ed. por Snow, Nancy y Taylor, Philip, 19-23, (Routledge, 2008). 
a course of actions, which [...] utilize the exchange of ideas, values, traditions and other aspects of culture or identity, whether to strengthen relationships, enhance socio-cultural cooperation, promote national interests and beyond ${ }^{6}$.

En esta definición se detalla que la diplomacia pública puede ser ejercida tanto por el sector público, como por el sector privado o la sociedad civil. Esto abre el espectro de qué tipo de actores comunicativos pueden participar en dicha actividad, incrementando tanto la variedad de mensajes emitidos como la riqueza del ecosistema comunicativo de una nación. En este sentido, podrían participar en la articulación de la diplomacia pública de una nación como Japón las diferentes instituciones del Estado, el Gobierno, empresas, creadores de contenido cultural (como películas, videojuegos, anime, manga y música), deportistas y líderes sociales y religiosos, entre otros.

Todos ellos participarían en la dimensión comunicativa de Japón, proyectando de forma multifacética la identidad nacional nipona en el escenario internacional. Esta articulación comunicativa se realizaría de forma bottom-up o ascendente, es decir, de abajo, desde la sociedad, hacia arriba, en contraste con una articulación top-down o descendente, en la cual la comunicación se realizaría desde el Gobierno hacia la sociedad (nacional o internacional).

En los extremos teóricos de este espectro, estas dos aproximaciones corresponderían a un sistema democrático en la articulación bottom-up y a un sistema no democrático en la articulación top-down. En esta asimilación, la existencia de libertad de expresión en una sociedad democrática permitiría que todos los actores comunicativos de la misma participasen en la diplomacia pública de la nación sin necesidad de una autorización o tutela gubernamental. En cambio, en los regímenes no democráticos, la libertad de expresión se encontraría restringida y toda la actividad comunicativa del país en la dimensión diplomática pública recaería bajo el auspicio gubernamental, con el evidente coste que esto implicaría, así como con la reducción de voces y variedad de las mismas que pueden producirse en este caso.

Por supuesto, estos dos extremos en este espectro de análisis serían de tipo teórico, puesto que en la realidad es posible encontrar un amplio número de espacios grises entre una teórica restricción absoluta de la libertad de expresión (en un sistema top-down puro) y una libertad absoluta de expresión (en un sistema bottom-up perfecto).

Si se aplicase una herramienta estadística para medir estos dos extremos teóricos, el coeficiente de Gini se revelaría como el indicador más adecuada para llevar a cabo esta medición. En esta aplicación, el coeficiente de Gini oscilaría entre dos extremos teóricos: cero o máxima igualdad (donde cada individuo tendría igual capacidad comunicativa) y uno o máxima desigualdad (donde la sociedad no tendría ninguna capacidad comunicativa y el Gobierno sería el único actor comunicativo. Como se ha indicado, estos dos extremos son meramente teóricos, al igual que ocurre con la aplicación más extendida del coeficiente de Gini: la desigualdad económica. La realidad práctica oscilaría entre un ecosistema comunicativo más plural y variado o uno menos plural y más jerarquizado. En esta categorización, Japón, como nación democrática que reconoce la libertad de expresión ${ }^{7}$, disfrutaría de una mayor variedad de actores comunicativos que participan en su diplomacia pública $^{8}$.

\section{b) Poder blando o soft power}

La idea de poder blando o soft power, en cambio, es diferente de la de diplomacia pública. Esta terminología fue acunada por Josep S. Nye en 2004 y es definida como "the ability of a nation to shape the preferences of other nation through diplomacy and attraction."

En esta consideración vemos que aquí se incluye diplomacia (tanto tradicional como pública) así como acciones de "atracción" realizada por una nación sobre otra. El objetivo no sería tanto una proyección internacional de la identidad de una nación, como el lograr una meta particular: influir en la jerarquía de preferencias de otra nación. En cualquier caso, estos dos conceptos están bastante relacionados.

En el caso de Japón, su diplomacia pública estaría constituida por todas las acciones de proyección comunicativa y cultural realizadas por los actores comunicativos nipones. El poder blando de Japón, en

\footnotetext{
Institute for Cultural Diplomacy, "Definition. What is Cultural Diplomacy?”, consultado el 2-6-2021, https:/www.culturaldiplomacy.org/index. php?en culturaldiplomacy

En la propia página web oficial del Gabinete del Primer Ministro de Japón se puede encontrar una traducción al inglés de la Constitución de Japón. En ella, su artículo 21 reconoce la libertad de expression junto a otros derechos y dice así: Freedom of assembly and association as well as speech, press and all other forms of expression are guaranteed. No censorship shall be maintained, nor shall the secrecy of any means of communication be violated. Prime Minister of Japan and His Cabinet, Japón, “The Constitution of Japan".

8 El coeficiente de Gini ha sido utilizado por investigaciones académicas para medir para analizar el proceso de popularización y democratización de las herramientas digitales de comunicación. Véase Martin Hilbert, "Technological Information Inequality as an Incessantly Moving Target: The Redistribution of Information and Communication Capacities Between 1986 and 2010”, Journal of the American Society for Information Science and Technology 65, no. 4, (2014): 821-835.

Joseph S. Nye, Soft Power: The Means To Success In World Politics, (New York, PublicAffairs: 2005).
} 
cambio, abarcaría su diplomacia pública junto con la diplomacia tradicional y cualquier acción para atraer a otras naciones hacia una jerarquía de preferencias determinada.

La idea de poder blando contrasta con la otra vertiente de poder: el poder duro o hard power que Nye define como la habilidad de una nación para generar en otras naciones un curso de acción deseado empleando medios militares y económicos. Ambos poderes se combinarían en lo que Nye denominó smart power o poder inteligente, que sería el uso de forma inteligente que una nación hace de sus capacidades de poder duro y poder blando. Es decir, saber emplear de forma adecuada tanto las herramientas de coacción, como de persuasión ${ }^{10}$.

Japón, al no poder disponer por el Artículo 9 de su Constitución de un ejército como tal y de haber renunciado a su derecho a la guerra ${ }^{11}$, sus herramientas para actuar internacionalmente se encontrarían limitadas ${ }^{12}$. Según esta categorización de Nye, Japón tendría solamente el poder blando y la parte económica del poder duro. Esto ha hecho que, para Japón, la adquisición y procesamiento de información e inteligencia relativa a su seguridad nacional sea de suma importancia, lo que ha llevado a la modernización de sus servicios de inteligencia ${ }^{13} \mathrm{y}$ a los reiterados intentos de crear un intercambio sólido de inteligencia militar con naciones potencialmente aliadas como es Corea del Sur ${ }^{14}$.

Dicha limitación ha sido aprovechada de forma exitosa por parte de Japón para convertirse en lo que algunos analistas han descrito como "un superpoder en términos de poder blando" ${ }^{15}$ fruto de su producción cultura ${ }^{16} \mathrm{y}$ tecnológica ${ }^{17}$ algo que se podrá ser explotado con los JJ. OO. de Tokio 2021 tal y como hizo con los de 1964.

En esta dimensión del poder blando y diplomacia pública o cultural, los Juegos Olímpicos de Tokio serían el mayor exponente de éstos, algo que enlaza con el nacimiento de los propios Juegos como instrumento político: ya en su origen, en el año 776 a.C., los JJ. OO. se revelaban como una herramienta diplomática de extraordinaria fortaleza al obligar a una tregua sagrada a las diferentes ciudades-estado que participaban en los mismos ${ }^{18}$. Dicha medida se observaba hasta el punto de que si alguna ciudad-estado violaba la tregua, sus atletas eran expulsados de la competición. Por lo tanto, desde su mismo origen fue posible lograr objetivos políticos internacionales empleando esta herramienta cultural. Esta misma manifestación se ha repetido en épocas más recientes, demostrando que los JJ. OO. han sido durante toda la historia un campo de batalla diplomático.

Los propios JJ. OO. de Tokio 1964 sufrieron también el boicot de varios países: Corea del Norte retiró a sus atletas justo antes de la inauguración oficial de los Juegos, mientras que China e Indonesia decidieron no participar. Las razones esgrimidas fueron que el COI impidió participar en estos JJ. OO. a los atletas que compitieron en los Games of the New Emerging Forces (GANEFO), una suerte de JJ. OO. alternativos organizados por Sukarno, Presidente de Indonesia. Los GANEFO querían servir como plataforma diplomática de lanzamiento de las llamadas "naciones emergentes", que eran Estados socialistas que se habían independizado recientemente ${ }^{19}$.

10 Aunque el término smart power ha aparecido en anteriores ocasiones empleado por diferentes autores con diferentes interpretaciones, en este artículo se empleará la taxonomía de Nye. Véase Joseph S. Nye, "Public Diplomacy and Soft Power", The Annals of the American Academy of Political and Social Science 616, Public Diplomacy in a Changing World, (2008): 94-106.

11 En la página web oficial del Gabinete del Primer Ministro de Japón aparece el Artículo 9 traducido así: Aspiring sincerely to an international peace based on justice and order, the Japanese people forever renounce war as a sovereign right of the nation and the threat or use of force as means of settling international disputes. In order to accomplish the aim of the preceding paragraph, land, sea, and air forces, as well as other war potential, will never be maintained. The right of belligerency of the state will not be recognized. Prime Minister of Japan and His Cabinet, Japón (2021): The Constitution of Japan, promulgada el 3 de noviembre de 1946, entrada en vigor el 3 de mayo de 1947, https://japan.kantei.go.jp/constitution_and_ government_of_japan/constitution_e.html

12 Carmen Tirado Robles, "Las recientes reformas legislativas en materia de política exterior y seguridad en Japón”. En Política exterior de Japón, Cuadernos Comillas de Política Exterior, no. 3, ed. por Javier Gil, 69-86, (Madrid: Universidad Pontificia Comillas, 2019).

13 Juan Luis López-Aranguren, "The Communicative Dimension and Security in Asia-Pacific: A communicative-viewing proposal for reform of the Japanese Intelligence Services”, UNISCI Discussion Papers 41, (Mayo 2016): 29-52.

14 Juan Luis López-Aranguren, "Seoul and Tokyo's new security axis: If you want peace... share intelligence", Asia \& The Pacific Policy Society Forum, (9-12-2017). https://www.policyforum.net/seoul-tokyos-new-security-axis/

15 Joshua Walker, “Japan is a soft power superpower", The Japan Times, (1-12-2019), https://www.japantimes.co.jp/opinion/2019/12/01/commentary/ japan-commentary/japan-soft-power-superpower/

16 Asger Røjle Christensen, “Cool Japan, Soft Power”, Global Asia 6, no. 1, (Marzo 2011).

17 Yee-Kuang Heng, "Beyond 'kawaii' pop culture: Japan's normative soft power as global trouble-shooter", The Pacific Review 27, no. 2, (2014): 169-192.

18 De hecho, la propia Naciones Unidas, como organización global que vela por la paz mundial, destaca en su página web la utilidad histórica de los JJOO en la paz sagrada o ekecheria, realizando a la vez una apelación a los valores olímpicos para unir a los pueblos abandonando los conflictos. United Nations, "The United Nations and the Olympic Truce", consultado el 2-6-2021, https://www.un.org/en/events/olympictruce/background. shtml

19 Sólo se realizaron dos ediciones de estos "JJOO alternativos", cada edición contando con menos participantes. La primera, en 1963, contó con la participación oficial de República Popular de China, la URSS, República Árabe Unida (antiguo Egipto), Indonesia, Corea del Norte, Argentina y Japón. El resto de las 51 naciones que participaron no enviaron a sus equipos nacionales por las sanciones del COI. La mayoría de los deportistas que participaron no pudieron clasificarse para los JJOO, por lo que los GANEFO se vieron como unos JJOO de segunda categoría. En la segunda edición, celebrada en 1967, este evento se renombró como “Asian GANEFO” y sólo participaron 17 naciones, incluyendo a Japón. En la tercera edición o segunda Asian GANEFO, Pekín, ciudad anfitriona, canceló su participación y los planes se trasladaron a Pyonyang, capital de Corea del Norte. Sin embargo, los sucesivos abandonos de los impulsores originales de GANEFO provocaron el colapso de esta organización y su desaparición como herramienta de diplomacia pública. Véase Ewa T. Pauker, “Ganefo I: Sports and Politics in Djakarta”, Asian Survey 5, no. 4, (1965): 171-185. 
De igual manera, en 1980 EE. UU. y otros 65 países boicotearon los JJ. OO. de Moscú en protesta por la invasión de Afganistán por parte de la URSS. Como represalia, la URSS y otros 13 Estados boicotearon la siguiente edición de los JJ. OO. en 1984, celebrada en Los Ángeles. Tal y como se puede comprobar, la dimensión olímpica es una extensión más de la diplomática.

\section{Japón como anfitriona de los JJ. OO.: De Tokio 1964 a Tokio 2021}

A continuación, se realizará una comparación entre la experiencia de los Juegos Olímpicos de Tokio 1964 y los Juegos Olímpicos de Tokio 2021 en varios niveles: geopolítico, interno japonés y comunicativo mediante la diplomacia olímpica.

\subsection{La diplomacia pública de los Juegos Olímpicos de Tokio de 1964}

Japón, por su parte, es una nación que ya tuvo experiencia como anfitriona en los JJ. OO. de Tokio de $1964^{20}$. En aquella ocasión, el mundo se encontraba divido por la Guerra Fría y 93 naciones participaron en los JJ. OO. de Tokio. Japón ya fue capaz de articular su diplomacia pública en esta celebración directamente con la elección del portador de la antorcha olímpica. El elegido para tal tarea fue el atleta Sakai Yoshinori, quien nació en Hiroshima el mismo día en que cayó la bomba atómica (6 de agosto de 1945). Sakai, como hibakusha o superviviente de los bombardeos atómicos, representaba la recuperación de Japón como nación asolada por la guerra, proyectando internacionalmente el deseo de Japón de superar los conflictos bélicos y construir una paz mundial.

Asimismo, Japón aprovechó esos JJ. OO. de 1964 para realizar también una intensa diplomacia pública mostrándose al mundo como nación líder en innovación tecnológica. Fruto de ello fue la inauguración del primer Shinkansen o tren bala de Japón ese mismo año ${ }^{21}$ y la construcción de la Torre de Tokio en 1958 para asegurar la cobertura en toda la zona de Kantō de las nuevas emisiones de televisión que, entre otras razones, se iban a producir con ocasión de estos JJ. OO. De hecho, la primera emisión televisiva en color para la audiencia general tuvo lugar con ocasión de dicha celebración.

En la actualidad, Japón sigue ejerciendo uno de los liderazgos indiscutibles en tecnología ferroviaria, aunque disputada por otras naciones que están dedicando una fuerte inversión a la innovación científica y tecnológica como China. La importancia que estas soluciones de transporte tienen para las sociedades modernas ha llevado a que estas sean unos de los intercambios más importantes que se han producido en las relaciones entre Japón y la India en el marco del Free and Open Indo-Pacific (FOIP) Strategy. En este intercambio, Japón está construyendo y financiando parcialmente el desarrollo de estaciones de metro en numerosas ciudades indias y la construcción de la línea de alta velocidad entre Bombay y Ahmedabad. La importancia que este tipo de colaboraciones tiene en el fortalecimiento de relaciones entre naciones permite a algunos autores hablar de una "diplomacia ferroviaria" que podríamos encuadrarla en la diplomacia pública de tipo tecnológico ${ }^{22}$.

\subsection{La diplomacia pública de los Juegos Olímpicos de Tokio de 2021}

Japón también ha sabido aprovechar la experiencia olímpica contemporánea como instrumento de diplomacia pública desde el primer momento en el que tuvo oportunidad de presentarse al mundo como nación anfitriona: en la ceremonia de clausura de los JJ. OO. de Río de Janeiro de 2016. En ella, el entonces Primer Ministro japonés, Abe Shinzo, realizó una performance ataviado como Mario Bros a la hora de recoger el testigo olímpico. Esto fue una buena muestra de que incluso cinco años antes del inicio oficial de los JJ. OO. de Tokio 2021 (retrasados por la pandemia del COVID-19), Japón ya supo aprovechar su proyección internacional como plataforma para ejercer su diplomacia pública.

Los JJ. OO. de Tokio han sido concebidos como una oportunidad histórica de reinvención del país a nivel interno y global tras la catástrofe de Fukushima causada por el Gran Terremoto del Este de Japón y el

20 La elección de Tokio como sede de los JJOO de 1964 fue rotunda en la 55 Sesión del Comité Olímpico Internacional celebrada en Múnich (República Federal Alemana en ese tiempo). Tokio se impuso con 34 votos a Detroit (10), Viena (9) y Bruselas (5). En ese sentido, se puede constatar que no solamente Japón estaba dispuesta a emitir su voz como actor de diplomacia pública en el mundo, sino que también el mundo estaba dispuesto a escuchar a Japón como símbolo internacional de reconstrucción y de paz tras la postguerra.

21 El Shinkansen o tren bala se convirtió en el símbolo de la excelencia tecnológica de Japón. La necesidad de unir las diferentes regiones japonesas que implicaba la rápida modernización de la economía se enfrentaba a la propia orografía del archipiélago japonés, con un 75\% de su territorio de tipo montañoso. El desarrollo y construcción de tecnología propia japonesa del Shinkansen catapultó a Japón, entre otros avances tecnológicos, como una de las naciones líderes mundiales en innovación tecnológica, convirtiéndose en uno de los símbolos del llamado milagro económico japonés. Véase Christopher Hood, Shinkansen: From Bullet Train to Symbol of Modern Japan, (New York: Routledge, 2006).

22 Shang-su Wu, “Japan's Train Diplomacy. Japan's railroad industry could become a significant tool of foreign policy”, The Diplomat, (9-11-2015), https://thediplomat.com/2015/11/japans-train-diplomacy/ 
consiguiente tsunami. Para ello, The Tokyo Oganising Committe of the Olympic and Paralympic Games ha publicado un documento oficial titulado Tokyo 2020 Action \& Legacy Plan 2016 ${ }^{23}$, en el cual se detallan los tres objetivos principales que Japón pretende alcanzar con la celebración de estos JJ. OO. Estos tres objetivos prioritarios son: la conexión entre la ciudadanía y los valores olímpicos, el refuerzo de la proyección cultural de Japón y el asegurar un legado para las generaciones futuras.

\subsubsection{Primer objetivo: fortalecer la conexión entre la ciudadanía y los valores olímpicos}

El primer objetivo es lograr una gran conexión entre el mayor número de ciudadanos y colectivos con el espíritu de los JJ. OO. Es posible observar aquí que la meta a lograr es fortalecer la cohesión social japonesa. Esta cohesión social puede verse como una manifestación de una estabilidad social confuciana que algunos antropólogos han identificado como un rasgo idiosincrático puro del carácter japonés ${ }^{24}$. Dicha estabilidad social puede actuar como una fuente de fuerza moral para la sociedad japonesa ante los retos tanto naturales (como el Gran Terremoto del Este de Japón o la pandemia del COVID-19), como artificiales (como la mayor asertividad geopolítica de China o la amenaza del programa nuclear de Corea del Norte).

Este primer objetivo es de tipo interno o doméstico. Como se puede comprobar aquí, la adopción de un rol internacional como potencia cultural y comunicativa con motivo de los JJ. OO. no solamente tiene un efecto en el campo de actuación (la dimensión comunicativa global) sino que también tiene un importante impacto en el propio actor comunicativo (en este caso la nación japonesa). El fortalecimiento del sentimiento de pertenencia a una determinada comunidad nacional y cultural con motivo de los JJ. OO. no solamente es una consecuencia de este evento, sino también un objetivo definido políticamente como una meta estratégica para Japón.

\subsubsection{Segundo objetivo: reforzar la proyección cultural de Japón}

El segundo objetivo introduce la dimensión exterior o internacional: maximizar la proyección cultural tanto nacional como global. Japón busca fortalecer también su capacidad comunicativa y su rol como actor en materia de diplomacia pública. El soft power de Japón, por lo tanto, se vería reforzado. Este fortalecimiento de su rol comunicativo incluiría diferentes dimensiones de Japón como potencia cultural y comunicativa. El turismo, por ejemplo, es uno de los campos más importantes de esta dimensión: Japón pretende aprovechar los JJ. OO. de Tokio para proyectar internacionalmente su atractivo turístico con el reclamo de su arte, historia, cultura y naturaleza para competir con otros destinos turísticos de Asia que están haciéndose más competitivos. Sabiendo que los JJ. OO. atraerán a millones de visitantes a Japón y que los mismos serán cubiertos por los medios de comunicación de todo el mundo, Japón quiere aprovechar este escaparate comunicativo internacional para incrementar su cuota de participación en el mercado turístico mundial, convirtiéndose en uno de los destinos preferidos para millones de turistas de todo el mundo una vez se levanten las restricciones de viaje por la pandemia.

También el liderazgo económico es otra de las dimensiones en las cuales la diplomacia pública de una nación puede manifestarse ${ }^{25}$. La percepción por parte de los consumidores de que los productos producidos en una nación o territorio son de alta calidad aporta indudables beneficios económicos a la economía de dicha nación.

En primer lugar, esta nación encuentra más fácil ocupar mayores nichos de mercado internacional al identificarse dicha nacionalidad con mayor calidad, por lo que desplazan a competidores que no disponen de esta reputación. En segundo lugar, los consumidores están dispuestos a pagar un precio más alto por dichos productos al asociarlos con una mayor calidad, lo que permite a los productores disponer de mayor financiación para incrementar sus procesos de innovación, invertir en maquinaria y expandirse a nuevos mercados. En tercer y último lugar, la reputación de un determinado tipo de producto puede trasladarse a otros bienes similares, lo que termina generando un círculo virtuoso que se ha denominado como "marca país" y que termina por beneficiar a todos los sectores de la economía de dicha nación. Japón, en este sentido, busca recuperar el liderazgo como nación puntera en tecnología para competir con otras economías asiáticas que han incrementado su competitividad al fabricar productos tecnológicos a bajo precio ${ }^{26}$. El fortalecimiento de

23 The Tokyo Organising Committee of the Olympic and Paralympic Games, "Tokyo 2020 Action \& Legacy Plan 2016. Participating in the Tokyo 2020 Games, Connecting with Tomorrow", (2016), https://gtimg.tokyo2020.org/image/upload/production/kndtqwos6ucxo4mfkpey.pdf

24 Estos antropólogos consideran que Japón es la nación que con mayor éxito ha implementado las prescripciones del confucianismo, entre las que destaca la piedad filial y, por extensión, el respeto a la autoridad, así como la paz social como bien supremo a proteger. Véase Kiri Paramore, Japanese Confucianism: A Cultural History, (Londres: Cambridge University Press, 2016).

25 Japón necesita incrementar su capacidad innovadora para mantener su competitividad frente a nuevos competidores asiáticos. López-Aranguren, Juan Luis, "El glocalismo como herramienta para potenciar la creatividad y la innovación de Japón en un mundo globalizado", MIRAI. Estudios Japoneses 4, (2020): 11-22.

26 Un campo en el que Japón quiere convertirse en referente mundial es el de la Inteligencia Artificial. Véase Ana Gascón Marcén, "Los principios para el desarrollo de la Inteligencia Artificial en Japón y las avenidas para la cooperación con la Unión Europea”, MIRAI. Estudios Japoneses 4, (2020): 23-34. 
la marca país de Japón permitiría a esta nación el incrementar su competitividad internacional, estimulando su economía nacional y ocupando nuevos nichos de mercado en un mundo cada vez más globalizado.

\subsubsection{Tercer objetivo: asegurar un legado a futuras generaciones.}

El tercer y último objetivo es asegurar un legado de valor a las futuras generaciones, tal y como se hizo con los JJ. OO. de Tokio 1964. Esto supone ya una apuesta por el futuro, pues no se contemplan los JJ. OO. sólo como una herramienta política para lograr un objetivo puntual de índole nacional o internacional, sino que se plantean también como un compromiso con las futuras generaciones para ofrecerles un legado tanto material como inmaterial que mejore las sociedades japonesa y global.

Estos tres objetivos del Tokyo 2020 Action \& Legacy Plan 2016 se han puesto en estrecha relación con el espíritu de recuperación nacional tras el Gran Terremoto del Este de Japón de 2011. De hecho, en el propio plan se indica que:

Following this period of high economic growth, Japan developed into a fully matured economy. However, with the earthquake and tsunami that ravaged Japan's Tohoku region in March 2011, Japan was once again faced with having to recover and reconstruct after a major disaster. Some 50 years after the 1964 Games, Japan is again confronted by such questions as how Tokyo and Japan should embrace the Olympic and Paralympic Games in 2020; how it should demonstrate its gratitude to the international community for the immense support and encouragement received from around the world in the aftermath of the 2011 disaster; and how to communicate the vital role that sport has played in the recovery efforts and its valuable contribution to society ${ }^{27}$.

Estos objetivos se han categorizado en cinco dimensiones diferentes para articularlos y proyectarlos tanto de forma doméstica como internacional a través de la acción comunicativa. La primera dimensión es deporte y salud, pues se entiende que la promoción de la práctica deportiva en la sociedad nipona e internacional con motivo de los JJ. OO. puede mejorar la calidad de vida y la salud de millones de participantes. En segundo lugar, la cultura y la educación. En este sentido, los JJ. OO. son un vehículo de transmisión de valores deportivos (sacrificio, deportividad, respeto, cooperación, esfuerzo, amistad) que pueden ayudar a construir una sociedad doméstica e internacional mejor, más pacífica y feliz. En tercer lugar, la planificación urbana y la sostenibilidad. En esta dimensión se entiende que los requerimientos logísticos y de infraestructuras de los JJ. OO. (tanto para los deportistas como para los espectadores y los profesionales que acudan a los mismos) supone una oportunidad para mejorar la planificación urbanística de Tokio y la sostenibilidad de Japón como nación concienciada con el medio ambiente. En cuarto lugar, la economía y la tecnología, buscando recuperar y fortalecer el liderazgo tecnológico de Japón. En quinto y último lugar, la recuperación del desastre de Fukushima o Gran Terremoto del Este de Japón.

\section{Metodología: Indicadores internacionales de la diplomacia pública de Japón}

Finalmente, es posible analizar y cuantificar mediante metodologías cuantitativas la capacidad de Japón para ejercer su labor como potencia de diplomacia pública en el mundo. Para ello, se recopilan diferentes indicadores de presencia y capacidad comunicativa global de cada nación, así como la percepción que logran en la audiencia global, con el objetivo de obtener una medida objetiva acerca de su impacto. Por supuesto, cualquier modelización de un campo tan complejo como es el de la diplomacia pública será simplemente una aproximación al fenómeno, pero al menos permite hacerse una idea sobre su influencia y establecer comparaciones entre los países.

Dos de estos indicadores son, por un lado, The Soft Power 30 (editado por Portland, Facebook y el USC Center for Public Diplomacy ${ }^{28}$; y el Global Soft Power Index, elaborado por la consultora Brand Finance. ${ }^{29}$

The Soft Power 30 actualiza anualmente la posición de cada una de las principales naciones en términos de poder blando con informes detallados sobre esta dimensión. ${ }^{30}$ En este indicador de 2019 (la última edición disponible en el momento de la publicación de este artículo) Japón ocupa la octava posición mundial obteniendo 75,71 puntos de un total de 100, por detrás de Francia, Reino Unido, Alemania, Suecia, Estados Unidos, Suiza y Canadá. Por comparación, Francia, la primera potencia en este indicador, obtendría 80,28 puntos de poder blando.

The Tokyo Organising Committee of the Olympic and Paralympic Games.

The Soft Power 30, "The Soft Power 30", (2019), https://softpower30.com/

Brand Finance, "Global Soft Power Index", (2021), https://brandirectory.com/globalsoftpower/

Portland, “The Soft Power 30. A Global Ranking of Soft Power 2019”, (2019), https://softpower30.com/wp-content/uploads/2019/10/The-Soft-

Power-30-Report-2019-1.pdf 
Este indicador se compone, asimismo, de siete variables o subíndices correspondientes a diferentes dimensiones o campos que participan en el poder blando o diplomacia pública de una nación, asignándose una puntuación a cada uno. Estos son: la dimensión digital (donde Japón obtiene 7 puntos), compañías (7), educación (16), cultural (6), conexión (5), gobierno (16) y sondeos (7).

Las seis primeros variables son extraídas de diferentes índices objetivos de la nación y tienen un peso en la puntuación final del 65\%. La última variable, sondeos, analiza la percepción de la audiencia global sobre la marca nación de Japón (y del resto de participantes en el estudio) y tiene un peso del 35\%. Esta dimensión de sondeos se compone, a su vez, de siete categorías: gastronomía, productos tecnológicos, amigabilidad, cultural, bienes de lujo, política exterior y calidad de vida. Estos sondeos se han realizado, en la última edición, sobre una muestra de 12.500 personas de 25 países de todo el mundo con una amplia representatividad. Al respecto, el informe destaca las fortalezas de Japón en poder blando:

Japan sees a noteworthy improvement in the Culture sub-index, jumping an impressive eight spots to reach 6th place. Hosting high-profile international events proved to be a blessing to Japan, indicating that Japan knows exactly how to leverage its wide-ranging cultural assets. The 2019 Rugby World Cup and G20 Summit were opportunities to build up and strengthen Japan's soft power reserves. With the 2020 Summer Olympic and Paralympic Games approaching, Japan has already garnered favourable conversation around its efforts to keep the games sustainable and eco-friendly. As sports diplomacy has proven to be a tremendously useful tool, we wait expectantly to see how Japan uses this event as a platform to project contemporary Japanese culture around the world with its penchant for ingenuity ${ }^{31}$.

Asimismo, realiza recomendaciones para mejorar estos indicadores aprovechando la nueva era Reiwa como un catalizador de este periodo de transformación interna e internacional, un momento que se une al de la celebración de los JJ. OO. de Tokio:

Japan has made significant headway in the international arena with strong performances in the Engagement and Culture sub-indices. However, poor performances in both the Government and Education sub-indices suggest that there is scope for significant gains with considered government intervention in these areas. Moreover, the conflict between internal political considerations and outward global perceptions of Japan is a risk that needs to be managed, as demonstrated by reactions to the resumption of commercial whaling. The new Reiwa era, which can be interpreted as "beautiful harmony", could be a timely catalyst for change ${ }^{32}$.

Según este indicador, desde el año anterior (2018) Japón habría descendido levemente en términos absolutos desde los 76,22 puntos, pero tres posiciones desde la quinta posición en ese año, lo que indica que este descenso en el ranking se debe más a una mejora del poder blando de los otros competidores que a un problema de Japón. Tomando en cuenta todo el histórico de datos disponibles, Japón habría incrementado su puntuación global desde el primer año en el que se realiza este registro (2015) al obtener ese año 66,86 puntos.

Por su parte, según Global Soft Power Index, Japón sería la segunda potencia mundial en 2021 en poder blando con una puntuación de 60,6, sólo por detrás de Alemania que obtiene 62,2. Japón habría avanzado dos posiciones globales, de la cuarta a la segunda, desde 2020. Las variables que se recogen en este indicador son: familiaridad (7,5 puntos en séptima posición mundial), influencia (5,6 en sexta posición), reputación (7,3 en cuarta posición), negocios y comercio ( 7 puntos en primera posición), gobernanza (5,3 en sexta posición), relaciones internacionales (5,7 en séptima), cultura y legado $(5,2$ en sexta), media y comunicación $(4,1$ en décima), educación y ciencia (7,5 en primera), población y valores (4,5 en duodécima), respuesta al COVID-19 ( 5,8 en segunda posición mundial) y red de influencia (logrando el $55 \%$ en sexta posición). Como se puede apreciar, este indicador mejora la posición de Japón como potencia de diplomacia pública o poder blando (asumiendo que ambos términos sean sinónimos) respecto al índice The Soft Power 30.

\section{Conclusiones}

Japón se ha convertido en una potencia mundial en diplomacia pública por méritos propios. La limitación constitucional en el uso de la dimensión militar en su poder duro ha podido tener un peso muy importante en el hecho de que Japón haya apostado tanto y de forma tan estratégica por potenciar su diplomacia pública y su poder blando para poder desarrollar su rol internacional en las últimas décadas.

\footnotetext{
The Soft Power 30

The Soft Power 30.
} 
Esto se refleja en los indicadores objetivos que existen sobre la diplomacia pública de Japón, al considerar que se encuentra en las primeras posiciones mundiales en términos de la misma (la segunda posición mundial en Global Soft Power Index y la octava según The Soft Power 30).

Los JJ. OO. de Tokio 2021 pueden ser una oportunidad para Japón de potenciar su diplomacia pública, como ya lo fueron los de Tokio 1964 que marcaron el nuevo rol de Japón como potencia económica, tecnológica y cultural mundial tras recuperarse de la Segunda Guerra Mundial. De la misma forma que el llamado milagro económico japonés tuvo su proyección oficial en estos JJ. OO. de Tokio de 1964, los actuales JJ. OO. de Tokio 2021 podrían convertirse en el equivalente del nuevo rol internacional de Japón, simbolizando asimismo la superación del desastre del Gran Terremoto del Este de Japón.

Este pistoletazo de salida se ha tenido que retrasar debido al impacto de la pandemia mundial del COVID-19, pero incluso a pesar de este obstáculo, los propios indicadores mundiales de diplomacia pública muestran que Japón también está fortaleciendo su posición en este ámbito sanitario al mostrarse al mundo como uno de los países que mejor lo han hecho en la adopción de medidas de contención del virus protegiendo su economía.

Si Japón consolidase esta tendencia, tendría la oportunidad de simbolizar mediante la diplomacia olímpica la superación de la crisis producida por el desastre del Gran Terremoto del Este de Japón, la adopción de un rol más asertivo a nivel internacional y la consolidación de un mayor dinamismo en una economía japonesa que necesita reinventarse para competir con nuevos competidos asiáticos. Por lo tanto, la nueva era Reiwa y los JJ. OO. de Tokio 2021 pueden ser la plataforma de lanzamiento de este nuevo rol nacional e internacional de Japón para el siglo XXI. Japón, de esta forma, tiene una oportunidad histórica para reinventarse, proyectando su identidad al mundo mediante su diplomacia pública como ya lo hizo en 1964.

\section{Bibliografía}

Barberán, Francisco, Introducción al Derecho Japonés Actual, Pamplona, Navarra: Aranzadi 2013.

Brand Finance, "Global Soft Power Index", (2021), https://brandirectory.com/globalsoftpower/

Christensen, Asger Røjle, “Cool Japan, Soft Power”, Global Asia 6, no. 1, (Marzo 2011), https://www.globalasia.org/ v6no1/feature/cool-japan-soft-power_asger-r\% $\mathrm{C} 3 \% \mathrm{~B} 8 \mathrm{jle}$-christensen

Cull, Nicholas J., "Public Diplomacy before Gullion. The Evolution of a Phrase". En Routledge Handbook of Public Diplomacy, ed. por Snow, Nancy y Taylor, Philip, 19-23, Routledge, 2008. https://doi.org/10.4324/9780203891520

Gascón Marcén, Ana, "Los principios para el desarrollo de la Inteligencia Artificial en Japón y las avenidas para la cooperación con la Unión Europea”, MIRAI. Estudios Japoneses 4, (2020): 23-34. https://doi.org/10.5209/mira.67233

Heng, Yee-Kuang, "Beyond 'kawaii' pop culture: Japan's normative soft power as global trouble-shooter", The Pacific Review 27, no. 2, (2014): 169-192, https://doi.org/10.1080/09512748.2014.882391

Hilbert, Martin, "Technological Information Inequality as an Incessantly Moving Target: The Redistribution of Information and Communication Capacities Between 1986 and 2010", Journal of the American Society for Information Science and Technology 65, no. 4, (2014): 821-835. https://doi.org/10.1002/asi.23020

Hood, Christopher, Shinkansen: From Bullet Train to Symbol of Modern Japan, New York: Routledge, 2006.

Institute for Cultural Diplomacy, "Definition. What is Cultural Diplomacy?", consultado el 2-6-2021, https://www. culturaldiplomacy.org/index.php?en_culturaldiplomacy

López-Aranguren, Juan Luis, "The Communicative Dimension and Security in Asia-Pacific: A communicative-viewing proposal for reform of the Japanese Intelligence Services", UNISCI Discussion Papers 41, (Mayo 2016): 29-52. [doi:10.5209/rev_RUNI.2016.n41.52673].

López-Aranguren, Juan Luis, "Rational Choice Theory and International Communication: A Proposal for a New Interpretation of Article 21 of the Japanese Constitution", Osaka University Law Review 64, (Febrero 2017): 111-134. [http://hdl.handle.net/11094/59680].

López-Aranguren, Juan Luis "Seoul and Tokyo's new security axis: If you want peace... share intelligence", Asia \& The Pacific Policy Society Forum, (9-12-2017). https://www.policyforum.net/seoul-tokyos-new-security-axis/

López-Aranguren, Juan Luis, "El glocalismo como herramienta para potenciar la creatividad y la innovación de Japón en un mundo globalizado", MIRAI. Estudios Japoneses 4, (2020): 11-22. https://doi.org/10.5209/mira.67540

Nye, Joseph S., Soft Power: The Means To Success In World Politics, New York, PublicAffairs: 2005.

Nye, Joseph S., "Public Diplomacy and Soft Power", The Annals of the American Academy of Political and Social Science 616, Public Diplomacy in a Changing World, (2008): 94-106.

Paramore, Kiri, Japanese Confucianism: A Cultural History, Londres: Cambridge University Press, 2016.

Pauker, Ewa T., "Ganefo I: Sports and Politics in Djakarta", Asian Survey 5, no. 4, (1965): 171-185. https://doi. org/10.2307/2642364

Portland, "The Soft Power 30. A Global Ranking of Soft Power 2019", (2019), https://softpower30.com/wp-content/ uploads/2019/10/The-Soft-Power-30-Report-2019-1.pdf

Prime Minister of Japan and His Cabinet, Japón, "The Constitution of Japan", promulgada el 3 de noviembre de 1946, entrada en vigor el 3 de mayo de 1947, consultado el 2-6-2021, https://japan.kantei.go.jp/constitution_and_ government_of_japan/constitution_e.html 
The Soft Power 30, "The Soft Power 30”, (2019), https://softpower30.com/

The Tokyo Organising Committee of the Olympic and Paralympic Games, "Tokyo 2020 Action \& Legacy Plan 2016. Participating in the Tokyo 2020 Games, Connecting with Tomorrow”, (2016), https:/gtimg.tokyo2020.org/image/ upload/production/kndtqwos6ucxo4mfkpey.pdf

Tirado Robles, Carmen, "Las recientes reformas legislativas en materia de política exterior y seguridad en Japón". En Política exterior de Japón, Cuadernos Comillas de Política Exterior, no. 3, ed. por Javier Gil, 69-86, (Madrid: Universidad Pontificia Comillas, 2019).

United Nations, "The United Nations and the Olympic Truce”, consultado el 2-6-2021, https://www.un.org/en/events/ olympictruce/background.shtml

Walker, Joshua, “Japan is a soft power superpower", The Japan Times, (1-12-2019), https://www.japantimes.co.jp/ opinion/2019/12/01/commentary/japan-commentary/japan-soft-power-superpower/

Wu, Shang-su, “Japan's Train Diplomacy. Japan's railroad industry could become a significant tool of foreign policy”, The Diplomat, (9-11-2015), https://thediplomat.com/2015/11/japans-train-diplomacy/ 